\title{
On Coupled Systems for Hilfer Fractional Differential Equations with Nonlocal Integral Boundary Conditions
}

\author{
Athasit Wongcharoen, ${ }^{1}$ Sotiris K. Ntouyas $\mathbb{D D}^{2,3}$ and Jessada Tariboon ${ }^{4}{ }^{4}$ \\ ${ }^{1}$ Department of Mechanical Engineering Technology, College of Industrial Engineering Technology, \\ King Mongkut's University of Technology North Bangkok, Bangkok 10800, Thailand \\ ${ }^{2}$ Department of Mathematics, University of Ioannina, 45110 Ioannina, Greece \\ ${ }^{3}$ Nonlinear Analysis and Applied Mathematics (NAAM)-Research Group, Department of Mathematices, Faculty of Science, \\ King Abdulaziz University, P.O. Box 80203, Jeddah 21589, Saudi Arabia \\ ${ }^{4}$ Intelligent and Nonlinear Dynamic Innovations Research Center, Department of Mathematics, Faculty of Applied Science, \\ King Mongkut's University of Technology North Bangkok, Bangkok 10800, Thailand
}

Correspondence should be addressed to Jessada Tariboon; jessada.t@sci.kmutnb.ac.th

Received 10 April 2020; Revised 24 May 2020; Accepted 11 June 2020; Published 13 July 2020

Guest Editor: Kottakkaran Sooppy Nisar

Copyright ( 92020 Athasit Wongcharoen et al. This is an open access article distributed under the Creative Commons Attribution License, which permits unrestricted use, distribution, and reproduction in any medium, provided the original work is properly cited.

In this paper, we study a coupled system involving Hilfer fractional derivatives with nonlocal integral boundary conditions. Existence and uniqueness results are obtained by applying Leray-Schauder alternative, Krasnoselskii's fixed point theorem, and Banach's contraction mapping principle. Examples illustrating our results are also presented.

\section{Introduction}

The theory of fractional differential equations has been widely used in pure mathematics and applications in the fields of physics, biology, and engineering. There are many interesting results for qualitative analysis and applications. We refer the interested reader, in fractional calculus, to the classical reference texts such as [1-7]. In the literature, there exist several different definitions of fractional integrals and derivatives, and the most popular of them are Riemann-Liouville, Caputo, and other less-known such as Hadamard fractional derivative and the Erdeyl-Kober fractional derivative. A generalization of derivatives of both Riemann-Liouville and Caputo was given by Hilfer in [8] as

$$
{ }^{H} D^{\alpha, \beta} u(t)=I^{\beta(n-\alpha)} D^{n} I^{(1-\beta)(n-\alpha)} u(t),
$$

where $n-1<\alpha<n, 0 \leq \beta \leq 1, t>a \geq 0$, and $D^{n}=\left(\mathrm{d}^{n} / \mathrm{d} t^{n}\right)$.

He named it as generalized fractional derivative of order $\alpha$ and a type $\beta$. Many authors call it the Hilfer fractional derivative. We notice that when $\beta=0$, the Hilfer fractional derivative corresponds to the Riemann-Liouville fractional derivative:

$$
{ }^{H} D^{\alpha, 0} u(t)=D^{n} I^{n-\alpha} u(t) .
$$

When $\beta=1$, the Hilfer fractional derivative corresponds to the Caputo fractional derivative:

$$
{ }^{H} D^{\alpha, 1} u(t)=I^{n-\alpha} D^{n} u(t) .
$$

Such derivative interpolates between the Riemann-Liouville and Caputo derivative. Some properties and applications of the Hilfer derivative are given in $[9,10]$ and the references cited therein.

Initial value problems involving Hilfer fractional derivatives were studied by several authors, see, for example [11-16] and references therein. Nonlocal boundary value problems for Hilfer fractional derivative were studied in [17].

To the best of our knowledge, there is no work carried out on systems of boundary value problems with Hilfer fractional derivative in the literature. This paper come to fill 
this gap. Thus, the objective of the present work is to introduce a new class of coupled systems of Hilfer-type fractional differential equations with nonlocal integral boundary conditions and develop the existence and uniqueness of solutions. In precise terms, we consider the following coupled system:

$$
\begin{cases}{ }^{H} D^{\alpha, \beta} x(t)=f(t, x(t), y(t)), & t \in[a, b], \\ { }^{H} D^{\alpha_{1}, \beta_{1}} y(t)=g(t, x(t), y(t)), & t \in[a, b], \\ x(a)=0, x(b)=\sum_{i=1}^{m} \theta_{i} I^{\varphi_{i}} y\left(\xi_{i}\right), & \\ y(a)=0, y(b)=\sum_{j=1}^{n} \zeta_{j} I^{\psi_{j}} x\left(z_{j}\right), & \end{cases}
$$

where ${ }^{H} D^{\alpha, \beta}$ and ${ }^{H} D^{\alpha_{1}, \beta_{1}}$ are the Hilfer fractional derivatives of orders $\alpha$ and $\alpha_{1}, 1<\alpha, \alpha_{1}<2$, and parameters $\beta$ and $\beta_{1}$, respectively, $0 \leq \beta, \beta_{1} \leq 1$, and $I^{\varphi_{i}}, I^{\psi_{j}}$ are the Riemann-Liouville fractional integrals of order $\varphi_{i}>0$ and $\psi_{j}>0$, respectively, the points $\xi_{i}, z_{j} \in[a, b], a \geq 0$, $f, g:[a, b] \times \mathbb{R} \times \mathbb{R} \longrightarrow \mathbb{R}$ are continuous functions and $\theta_{i}$, $\zeta_{j} \in \mathbb{R}, \quad i=1,2, \ldots, m, \quad j=1,2, \ldots, n$ are given real constants.

The paper is organized as follows. We present our main results in Section 3, by applying Leray-Schauder alternative, Krasnoselskii's fixed point theorem, and Banach's contraction mapping principle, while Section 2 contains some preliminary concepts related to our problem. Examples are constructed to illustrate the main results.

\section{Preliminaries}

In this section, we introduce some notations and definitions of fractional calculus and present preliminary results needed in our proofs later $[2,5]$.

Definition 1. The Riemann-Liouville fractional integral of order $\alpha>0$ of a continuous function $u:[a, \infty) \longrightarrow \mathbb{R}$ is defined by

$$
I^{\alpha} u(t)=\frac{1}{\Gamma(\alpha)} \int_{a}^{t}(t-s)^{\alpha-1} u(s) \mathrm{d} s
$$

provided the right-hand side exists on $(a, \infty)$.

Definition 2. The Riemann-Liouville fractional derivative of order $\alpha>0$ of a continuous function is defined by

$$
{ }^{\mathrm{RL}} D^{\alpha} u(t):=D^{n} I^{n-\alpha} u(t)=\frac{1}{\Gamma(n-\alpha)}\left(\frac{\mathrm{d}}{\mathrm{d} t}\right)^{n} \int_{a}^{t}(t-s)^{n-\alpha-1} u(s) \mathrm{d} s
$$

where $n=[\alpha]+1,[\alpha]$ denotes the integer part of real number $\alpha$, provided the right-hand side is pointwise defined on $(a, \infty)$.

Definition 3. The Caputo fractional derivative of order $\alpha>0$ of a continuous function is defined by

$$
\begin{aligned}
{ }^{C} D^{\alpha} u(t):= & I^{n-\alpha} D^{n} u(t)=\frac{1}{\Gamma(n-\alpha)} \int_{a}^{t}(t-s)^{n-\alpha-1}\left(\frac{\mathrm{d}}{\mathrm{d} s}\right)^{n} \\
& \cdot u(s) \mathrm{d} s, \quad n-1<\alpha<n,
\end{aligned}
$$

provided the right-hand side is pointwise defined on $(a, \infty)$.

Lemma 1 (see [18]). If $g \in C^{n}[a, b], n-1<\alpha<n$, and $0 \leq \beta \leq 1$, then

(1) $I^{\alpha H} D^{\alpha, \beta} g(t)=g(t)-\sum_{k=1}^{n}\left((t-a)^{\gamma-k} / \Gamma(\gamma-k+1)\right)$ $(d / d t)^{n-k} I^{(1-\beta)(n-\alpha)} g(a)$

(2) ${ }^{H} D^{\alpha, \beta} I^{\alpha} g(t)=g(t)$

The following lemma deals with a linear variant of problem (4).

Lemma 2. Let $\varphi_{i}, \psi_{j}>0, \xi_{i}, z_{j} \in[a, b], a \geq 0, \theta_{i}, \zeta_{j} \in \mathbb{R}$, $i=1,2, \ldots, m, \quad j=1,2, \ldots, n, \quad 1<\alpha, \alpha_{1}<2, \quad 0 \leq \beta, \beta_{1} \leq 1$, $\gamma=\alpha+2 \beta-\alpha \beta, \quad \gamma_{1}=\alpha_{1}+2 \beta_{1}-\alpha_{1} \beta_{1}, h, h_{1} \in C([a, b], \mathbb{R})$, and

$$
\begin{aligned}
\Lambda= & \frac{(b-a)^{\gamma+\gamma_{1}-2}}{\Gamma(\gamma) \Gamma\left(\gamma_{1}\right)}-\left(\sum_{i=1}^{m} \theta_{i} \frac{\left(\xi_{i}-a\right)^{\gamma_{1}+\varphi_{i}-1}}{\Gamma\left(\gamma_{1}+\varphi_{i}\right)}\right) \\
& \cdot\left(\sum_{j=1}^{n} \zeta_{j} \frac{\left(z_{j}-a\right)^{\gamma+\psi_{j}-1}}{\Gamma\left(\gamma+\psi_{j}\right)}\right) \neq 0 .
\end{aligned}
$$

Then, the system

$$
\begin{cases}{ }^{H} D^{\alpha, \beta} x(t)=h(t), & t \in[a, b], \\ { }^{H} D^{\alpha_{1}, \beta_{1}} y(t)=h_{1}(t), & t \in[a, b], \\ x(a)=0 & \\ x(b)=\sum_{i=1}^{m} \theta_{i} I^{\varphi_{i}} y\left(\xi_{i}\right), & \\ y(a)=0 & \\ y(b)=\sum_{j=1}^{n} \zeta_{j} I^{\psi_{j}} x\left(z_{j}\right),\end{cases}
$$

is equivalent to the following integral equations: 


$$
\begin{aligned}
x(t)= & I^{\alpha} h(t)+\frac{(t-a)^{\gamma-1}}{\Lambda \Gamma(\gamma)}\left[\frac{(b-a)^{\gamma_{1}-1}}{\Gamma\left(\gamma_{1}\right)}\left(\sum_{i=1}^{m} \theta_{i} I^{\alpha_{1}+\varphi_{i}} h_{1}\left(\xi_{i}\right)-I^{\alpha} h(b)\right)\right. \\
& \left.+\left(\sum_{i=1}^{m} \theta_{i} \frac{\left(\xi_{i}-a\right)^{\gamma_{1}+\varphi_{i}-1}}{\Gamma\left(\gamma_{1}+\varphi_{i}\right)}\right)\left(\sum_{j=1}^{n} \zeta_{j} I^{\alpha+\psi_{j}} h\left(z_{j}\right)-I^{\alpha_{1}} h_{1}(b)\right)\right] \\
y(t)= & I^{\alpha_{1}} h_{1}(t)+\frac{(t-a)^{\gamma_{1}-1}}{\Lambda \Gamma\left(\gamma_{1}\right)}\left[\frac{(b-a)^{\gamma-1}}{\Gamma(\gamma)}\left(\sum_{j=1}^{n} \zeta_{j} I^{\alpha+\psi_{j}} h\left(z_{j}\right)-I^{\alpha_{1}} h_{1}(b)\right)\right. \\
& \left.+\left(\sum_{j=1}^{n} \zeta_{j} \frac{\left(z_{j}-a\right)^{\gamma+\psi_{j}-1}}{\Gamma\left(\gamma+\psi_{j}\right)}\right)\left(\sum_{i=1}^{m} \theta_{i} I^{\alpha_{1}+\varphi_{i}} h_{1}\left(\xi_{i}\right)-I^{\alpha} h(b)\right)\right] .
\end{aligned}
$$

Proof. Operating fractional integral $I^{\alpha}$ on both sides of the first equation in (9) and using Lemma 1, we obtain

$$
x(t)-\sum_{k=1}^{2} \frac{(t-a)^{\gamma-k}}{\Gamma(\gamma-k+1)}\left(\frac{\mathrm{d}}{\mathrm{d} t}\right)^{2-k} I^{(1-\beta)(2-\alpha)} x(a)=I^{\alpha} h(t) .
$$

Then, we have, since $(1-\beta)(2-\alpha)=2-\gamma$,

$$
\begin{aligned}
x(t)= & \left.\frac{(t-a)^{\gamma-1}}{\Gamma(\gamma)}\left(\frac{d}{d t}\right) I^{2-\gamma} x(t)\right|_{t=a}+\left.\frac{(t-a)^{\gamma-2}}{\Gamma(\gamma-1)} I^{2-\gamma} x(t)\right|_{t=a} \\
& +I^{\alpha} h(t) \\
= & \left.\frac{(t-a)^{\gamma-1}}{\Gamma(\gamma)}{ }^{\gamma-1, \beta} x(t)\right|_{t=a}+\left.\frac{(t-a)^{\gamma-2}}{\Gamma(\gamma-1)} I^{2-\gamma} x(t)\right|_{t=a} \\
& +I^{\alpha} h(t) .
\end{aligned}
$$

Then,

$$
x(t)=\frac{c_{1}}{\Gamma(\gamma)}(t-a)^{\gamma-1}+\frac{c_{2}}{\Gamma(\gamma-1)}(t-a)^{\gamma-2}+I^{\alpha} h(t),
$$

where

$$
\begin{aligned}
& c_{1}=\left.{ }^{H} D^{\gamma-1, \beta} x(t)\right|_{t=a}, \\
& c_{2}=\left.I^{2-\gamma} x(t)\right|_{t=a} .
\end{aligned}
$$

By a similar way, we obtain

$$
y(t)=\frac{d_{1}}{\Gamma\left(\gamma_{1}\right)}(t-a)^{\gamma_{1}-1}+\frac{d_{2}}{\Gamma\left(\gamma_{1}-1\right)}(t-a)^{\gamma_{1}-2}+I^{\alpha_{1}} h_{1}(t) .
$$

By setting

$$
\begin{aligned}
& d_{1}=\left.{ }^{H} D^{\gamma_{1}-1, \beta} y(t)\right|_{t=a}, \\
& d_{2}=\left.I^{2-\gamma} y(t)\right|_{t=a},
\end{aligned}
$$

from the boundary conditions $x(a)=0$ and $y(a)=0$, we obtain $c_{2}=0$ and $d_{2}=0$. Then, we obtain

$$
\begin{aligned}
& x(t)=\frac{c_{1}}{\Gamma(\gamma)}(t-a)^{\gamma-1}+I^{\alpha} h(t), \\
& y(t)=\frac{d_{1}}{\Gamma\left(\gamma_{1}\right)}(t-a)^{\gamma_{1}-1}+I^{\alpha_{1}} h_{1}(t),
\end{aligned}
$$

$$
\sum_{i=1}^{m} \theta_{i} I^{\varphi_{i}} y\left(\xi_{i}\right)=d_{1} \sum_{i=1}^{m} \frac{\theta_{i}\left(\xi_{i}-a\right)^{\gamma_{1}+\varphi_{i}-1}}{\Gamma\left(\gamma_{1}+\varphi_{i}\right)}+\sum_{i=1}^{m} \theta_{i} I^{\alpha_{1}+\varphi_{i}} h_{1}\left(\xi_{i}\right),
$$$$
\sum_{j=1}^{n} \zeta_{i} I^{\psi_{i}} x\left(z_{j}\right)=c_{1} \sum_{j=1}^{n} \frac{\zeta_{j}\left(z_{j}-a\right)^{\gamma+\psi_{j}-1}}{\Gamma\left(\gamma+\psi_{j}\right)}+\sum_{j=1}^{n} \zeta_{j} I^{\alpha+\psi_{j}} h\left(z_{j}\right) .
$$

From $x(b)=\sum_{i=1}^{m} \theta_{i} I^{\varphi_{i}} y\left(\xi_{i}\right)$ and $y(b)=\sum_{j=1}^{n} \zeta_{j} I^{\psi_{j}}$ $x\left(z_{j}\right)$, we have

$$
\begin{aligned}
c_{1}= & \frac{1}{\Lambda}\left[\frac{(b-a)^{\gamma_{1}-1}}{\Gamma\left(\gamma_{1}\right)}\left(\sum_{i=1}^{m} \theta_{i} I^{\alpha_{1}+\varphi_{i}} h_{1}\left(\xi_{i}\right)-I^{\alpha} h(b)\right)\right. \\
& \left.+\left(\sum_{i=1}^{m} \theta_{i} \frac{\left(\xi_{i}-a\right)^{\gamma_{1}+\varphi_{i}-1}}{\Gamma\left(\gamma_{1}+\varphi_{i}\right)}\right)\left(\sum_{j=1}^{n} \zeta_{j} I^{\alpha+\psi_{j}} h\left(z_{j}\right)-I^{\alpha_{1}} h_{1}(b)\right)\right], \\
d_{1}= & \frac{1}{\Lambda}\left[\frac{(b-a)^{\gamma-1}}{\Gamma(\gamma)}\left(\sum_{j=1}^{n} \zeta_{j} I^{\alpha+\psi_{j}} h\left(z_{j}\right)-I^{\alpha_{1}} h_{1}(b)\right)\right. \\
& \left.+\left(\sum_{j=1}^{n} \zeta_{j} \frac{\left(z_{j}-a\right)^{\gamma+\psi_{j}-1}}{\Gamma\left(\gamma+\psi_{j}\right)}\right)\left(\sum_{i=1}^{m} \theta_{i} I^{\alpha_{1}+\varphi_{i}} h_{1}\left(\xi_{i}\right)-I^{\alpha} h(b)\right)\right] .
\end{aligned}
$$

Substituting the values of $c_{1}$ and $d_{1}$ in (18), we obtain solutions (10) and (11). The converse follows by direct computation. This completes the proof.

\section{Main Results}

Let $\mathscr{C}=C([a, b], \mathbb{R}), a \geq 0$, denote the Banach space of all continuous functions from $[a, b]$ to $\mathbb{R}$. The space $X=\left\{x: x(t) \in C^{2}([a, b], \mathbb{R})\right\}$ endowed with the norm $\|x\|=$ $\sup \{|x(t)|, \quad t \in[a, b]\} \quad$ is a Banach space. Let $Y=\left\{y:|y(t)| \in C^{2}([a, b], \mathbb{R})\right\} \quad$ with the norm $\|y\|=\sup \{|y(t)|, \quad t \in[a, b]\}$. It is obvious that the product 
space $(X \times Y,\|(x, y)\|)$ is Banach space with the norm $\|(x, y)\|=\|x\|+\|y\|$.

In view of Lemma 2 , we define two operators $\mathscr{K}: X \times$ $Y \longrightarrow X \times Y$ by

$$
\mathscr{K}(x, y)(t)=\left(\begin{array}{l}
\mathscr{K}_{1}(x, y)(t) \\
\mathscr{K}_{2}(x, y)(t)
\end{array}\right),
$$

where

$$
\begin{aligned}
\mathscr{K}_{1}(x, y)(t)= & I^{\alpha} f_{x, y}(t)+\frac{(t-a)^{\gamma-1}}{\Lambda \Gamma(\gamma)}\left[\frac{(b-a)^{\gamma_{1}-1}}{\Gamma\left(\gamma_{1}\right)}\left(\sum_{i=1}^{m} \theta_{i} I^{\alpha_{1}+\varphi_{i}} g_{x, y}\left(\xi_{i}\right)-I^{\alpha} f_{x, y}(b)\right)\right. \\
& \left.+\left(\sum_{i=1}^{m} \theta_{i} \frac{\left(\xi_{i}-a\right)^{\gamma_{1}+\varphi_{i}-1}}{\Gamma\left(\gamma_{1}+\varphi_{i}\right)}\right)\left(\sum_{j=1}^{n} \zeta_{j} I^{\alpha+\psi_{j}} f_{x, y}\left(z_{j}\right)-I^{\alpha_{1}} g_{x, y}(b)\right)\right], \\
\mathscr{K}_{2}(x, y)(t)= & I^{\alpha_{1}} g_{x, y}(t)+\frac{(t-a)^{\gamma-1}}{\Lambda \Gamma(\gamma)}\left[\frac{(b-a)^{\gamma_{1}-1}}{\Gamma\left(\gamma_{1}\right)}\left(\sum_{j=1}^{n} \zeta_{j} I^{\alpha+\psi_{j}} f_{x, y}\left(z_{j}\right)-I^{\alpha_{1}} g_{x, y}(b)\right)\right. \\
& \left.+\left(\sum_{j=1}^{n} \zeta_{j} \frac{\left(z_{j}-a\right)^{\gamma+\psi_{j}-1}}{\Gamma\left(\gamma+\psi_{j}\right)}\right)\left(\sum_{i=1}^{m} \theta_{i} I^{\alpha_{1}+\varphi_{i}} g_{x, y}\left(\xi_{i}\right)-I^{\alpha} f_{x, y}(b)\right)\right],
\end{aligned}
$$

where

$$
\begin{aligned}
& f_{x, y}(t)=f(t, x(t), y(t)), \\
& g_{x, y}(t)=g(t, x(t), y(t)), \quad t \in[a, b] .
\end{aligned}
$$

For computational convenience, we set

$$
\begin{aligned}
M_{1}= & \frac{(b-a)^{\alpha}}{\Gamma(\alpha+1)}+\frac{(b-a)^{\gamma-1}}{|\Lambda| \Gamma(\gamma)}\left[\frac{(b-a)^{\gamma_{1}+\alpha-1}}{\Gamma\left(\gamma_{1}\right) \Gamma(\alpha+1)}\right. \\
& \left.+\left(\sum_{i=1}^{m}\left|\theta_{i}\right| \frac{\left(\xi_{i}-a\right)^{\gamma_{1}+\varphi_{i}-1}}{\Gamma\left(\gamma_{1}+\varphi_{i}\right)}\right)\left(\sum_{j=1}^{n}\left|\zeta_{j}\right| \frac{\left(z_{j}-a\right)^{\alpha+\psi_{j}}}{\Gamma\left(\alpha+\psi_{j}+1\right)}\right)\right] \\
M_{2}= & \frac{(b-a)^{\gamma-1}}{|\Lambda| \Gamma(\gamma)}\left[\frac{(b-a)^{\gamma-1}}{\Gamma\left(\gamma_{1}\right)}\left(\sum_{i=1}^{m}\left|\theta_{i}\right| \frac{\left(\xi_{i}-a\right)^{\alpha_{1}+\varphi_{i}}}{\Gamma\left(\alpha_{1}+\varphi_{i}+1\right)}\right)\right. \\
& \left.+\frac{(b-a)^{\alpha_{1}}}{\Gamma\left(\alpha_{1}+1\right)}\left(\sum_{i=1}^{m}\left|\theta_{i}\right| \frac{\left(\xi_{i}-a\right)^{\gamma_{1}+\varphi_{i}-1}}{\Gamma\left(\gamma_{1}+\varphi_{i}\right)}\right)\right] \\
M_{3}= & \frac{(b-a)^{\gamma-1}}{|\Lambda| \Gamma\left(\gamma_{1}\right)}\left[\frac{(b-a)^{\gamma-1}}{\Gamma(\gamma)}\left(\sum_{j=1}^{n}\left|\zeta_{j}\right| \frac{\left(z_{j}-a\right)^{\alpha+\psi_{j}}}{\Gamma\left(\alpha+\psi_{j}+1\right)}\right)\right. \\
& \left.+\frac{(b-a)^{\alpha}}{\Gamma\left(\alpha_{1}+1\right)}\left(\sum_{i=1}^{m}\left|\zeta_{j}\right| \frac{\left(z_{j}-a\right)^{\gamma+\psi_{j}-1}}{\Gamma\left(\gamma+\psi_{j}\right)}\right)\right] \\
M_{4}= & \frac{(b-a)^{\alpha_{1}}}{\Gamma\left(\alpha_{1}+1\right)}+\frac{(b-a)^{\gamma_{1}-1}}{|\Lambda| \Gamma\left(\gamma_{1}\right)}\left[\frac{(b-a)^{\gamma+\alpha_{1}-1}}{\Gamma(\gamma) \Gamma\left(\alpha_{1}+1\right)}\right. \\
+ & \left.\left(\sum_{j=1}^{n}\left|\zeta_{j}\right| \frac{\left(z_{j}-a\right)^{\gamma+\psi_{j}-1}}{\Gamma\left(\gamma+\psi_{j}\right)}\right)\left(\sum_{i=1}^{m}\left|\theta_{i}\right| \frac{\left(\xi_{i}-a\right)^{\alpha_{1}+\varphi_{i}}}{\Gamma\left(\alpha_{1}+\varphi_{i}+1\right)}\right)\right]
\end{aligned}
$$

Banach's contraction mapping principle is applied in the first result to prove existence and uniqueness of solutions of system (4).

Theorem 1. Suppose that $f, g:[a, b] \times \mathbb{R} \times \mathbb{R} \longrightarrow \mathbb{R}$ are continuous functions. In addition, we assume that

$\left(H_{1}\right)$ There exist constants $\ell_{i}, n_{i}, i=1,2$, such that, for all $t \in[a, b]$ and $x_{i}, y_{i} \in \mathbb{R}, i=1,2$,

$$
\begin{aligned}
& \left|f\left(t, x_{1}, y_{1}\right)-f\left(t, x_{2}, y_{2}\right)\right| \leq l_{1}\left|x_{1}-x_{2}\right|+l_{2}\left|y_{1}-y_{2}\right| \\
& \left|g\left(t, x_{1}, y_{1}\right)-g\left(t, x_{2}, y_{2}\right)\right| \leq n_{1}\left|x_{1}-x_{2}\right|+n_{2}\left|y_{1}-y_{2}\right| .
\end{aligned}
$$

Then, system (4) has a unique solution on $[a, b]$, if

$$
\left(M_{1}+M_{3}\right)\left(\ell_{1}+\ell_{2}\right)+\left(M_{2}+M_{4}\right)\left(n_{1}+n_{2}\right)<1 \text {. }
$$

Proof. Define $\sup _{t \in[a, b]} f(t, 0,0)=N_{1}<\infty \quad$ and $\sup _{t \in[a, b]} g(t, 0,0)=N_{2}<\infty$ such that

$$
r>\frac{\left(M_{1}+M_{3}\right) N_{1}+\left(M_{2}+M_{4}\right) N_{2}}{1-\left[\left(M_{1}+M_{3}\right)\left(\ell_{1}+\ell_{2}\right)+\left(M_{2}+M_{4}\right)\left(n_{1}+n_{2}\right)\right]} .
$$

Now, we will show that the set $\mathscr{K} B_{r} \subset B_{r}$, where $B_{r}=\{(x, y) \in X \times Y:\|(x, y)\| \leq r\}$. For any $(x, y) \in B_{r}$, $t \in[a, b]$, we find that

$$
\begin{aligned}
|f(t, x(t), y(t))| & =|f(t, x(t), y(t))-f(t, 0,0)+f(t, 0,0)| \\
& \leq|f(t, x(t), y(t))-f(t, 0,0)|+|f(t, 0,0)| \\
& \leq \ell_{1}\|x\|+\ell_{2}\|y\|+N_{1}, \\
|g(t, x(t), y(t))| & \leq n_{1}\|x\|+n_{2}\|y\|+N_{2} .
\end{aligned}
$$




$$
\begin{aligned}
& \left|\mathscr{K}_{1}(x, y)(t)\right| \\
& \leq I^{\alpha}\left|f_{x, y}\right|(b)+\frac{(b-a)^{\gamma-1}}{|\Lambda| \Gamma(\gamma)}\left[\frac{(b-a)^{\gamma_{1}-1}}{\Gamma\left(\gamma_{1}\right)}\left(\sum_{i=1}^{m}\left|\theta_{i}\right| I^{\alpha_{1}+\varphi_{i}}\left|g_{x, y}\right|\left(\xi_{i}\right)+I^{\alpha}\left|f_{x, y}\right|(b)\right)\right. \\
& \left.+\left(\sum_{i=1}^{m}\left|\theta_{i}\right| \frac{\left(\xi_{i}-a\right)^{\gamma_{1}+\varphi_{i}-1}}{\Gamma\left(\gamma_{1}+\varphi_{i}\right)}\right)\left(\sum_{j=1}^{n}\left|\zeta_{j}\right| I^{\alpha+\psi_{j}}\left|f_{x, y}\right|\left(z_{j}\right)+I^{\alpha_{1}}\left|g_{x, y}\right|(b)\right)\right] \\
& \leq I^{\alpha}\left(\left|f_{x, y}-f_{0,0}\right|+\left|f_{0,0}\right|\right)(b)+\frac{(b-a)^{\gamma-1}}{|\Lambda| \Gamma(\gamma)}\left[\frac{(b-a)^{\gamma_{1}-1}}{\Gamma\left(\gamma_{1}\right)}\right. \\
& \times\left(\sum_{i=1}^{m}\left|\theta_{i}\right| I^{\alpha_{1}+\varphi_{i}}\left(\left|g_{x, y}-g_{0,0}\right|+\left|g_{0,0}\right|\right)\left(\xi_{i}\right)+I^{\alpha}\left(\left|f_{x, y}-f_{0,0}\right|\right)+\left|f_{0,0}\right|(b)\right) \\
& +\left(\sum_{i=1}^{m}\left|\theta_{i}\right| \frac{\left(\xi_{i}-a\right)^{\gamma_{1}+\varphi_{i}-1}}{\Gamma\left(\gamma_{1}+\varphi_{i}\right)}\right)\left(\sum_{j=1}^{n}\left|\zeta_{j}\right| I^{\alpha+\psi_{j}}\left(\left|f_{x, y}-f_{0,0}\right|+\left|f_{0,0}\right|\right)\left(z_{j}\right)\right. \\
& \left.\left.+I^{\alpha_{1}}\left(\left|g_{x, y}-g_{0,0}\right|\right)+\left|g_{0,0}\right|(b)\right)\right] \\
& \leq I^{\alpha}\left(\ell_{1}\|x\|+\ell_{2}\|y\|+N_{1}\right)(b)+\frac{(b-a)^{\gamma-1}}{|\Lambda| \Gamma(\gamma)}\left[\frac{(b-a)^{\gamma_{1}-1}}{\Gamma\left(\gamma_{1}\right)}\right. \\
& \times\left(\sum_{i=1}^{m}\left|\theta_{i}\right| I^{\alpha_{1}+\varphi_{i}}\left(n_{1}\|x\|+n_{2}\|y\|+N_{2}\right)\left(\xi_{i}\right)+I^{\alpha}\left(\ell_{1}\|x\|+\ell_{2}\|y\|+N_{1}\right)(b)\right) \\
& +\left(\sum_{i=1}^{m}\left|\theta_{i}\right| \frac{\left(\xi_{i}-a\right)^{\gamma_{1}+\varphi_{i}-1}}{\Gamma\left(\gamma_{1}+\varphi_{i}\right)}\right)\left(\sum_{j=1}^{n}\left|\zeta_{j}\right| I^{\alpha+\psi_{j}}\left(\ell_{1}\|x\|+\ell_{2}\|y\|+N_{1}\right)\left(z_{j}\right)\right. \\
& \left.\left.+I^{\alpha_{1}}\left(n_{1}\|x\|+n_{2}\|y\|+N_{2}\right)(b)\right)\right] \\
& \leq M_{1}\left(\ell_{1}\|x\|+\ell_{2}\|y\|+N_{1}\right)+M_{2}\left(n_{1}\|x\|+n_{2}\|y\|+N_{2}\right) \\
& =\left(M_{1} \ell_{1}+M_{2} n_{1}\right)\|x\|+\left(M_{1} \ell_{2}+M_{2} n_{2}\right)\|y\|+M_{1} N_{1}+M_{2} N_{2} \\
& \leq\left[M_{1}\left(\ell_{1}+\ell_{2}\right)+M_{2}\left(n_{1}+n_{2}\right)\right] r+M_{1} N_{1}+M_{2} N_{2} \text {. }
\end{aligned}
$$

Hence,

$$
\left\|\mathscr{K}_{2}(x, y)\right\| \leq\left[M_{3}\left(\ell_{1}+\ell_{2}\right)+M_{4}\left(n_{1}+n_{2}\right)\right] r+M_{3} N_{1}+M_{4} N_{2} .
$$

$$
\begin{aligned}
\left\|\mathscr{K}_{1}(x, y)\right\| \leq & {\left[M_{1}\left(\ell_{1}+\ell_{2}\right)+M_{2}\left(n_{1}+n_{2}\right)\right] r+M_{1} N_{1} } \\
& +M_{2} N_{2} .
\end{aligned}
$$

Similarly, we have

$$
\begin{gathered}
\left|\mathscr{K}_{2}(x, y)(t)\right| \leq M_{3}\left(\ell_{1}\|x\|+\ell_{2}\|y\|+N_{1}\right)+M_{4} \\
\cdot\left(n_{1}\|x\|+n_{2}\|y\|+N_{2}\right),
\end{gathered}
$$

and hence
Consequently, it follows that

$$
\begin{aligned}
\|\mathscr{K}(x, y)\| \leq & {\left[M_{1}\left(\ell_{1}+\ell_{2}\right)+M_{2}\left(n_{1}+n_{2}\right)\right] r+M_{1} N_{1}+M_{2} N_{2} } \\
& +\left[M_{3}\left(\ell_{1}+\ell_{2}\right)+M_{4}\left(n_{1}+n_{2}\right)\right] r+M_{3} N_{1}+M_{4} N_{2} \leq r,
\end{aligned}
$$

which implies $\mathscr{K} B_{r} \subset B_{r}$. Next, we will show that the operator $\mathscr{K}$ is a contraction mapping. For any $\left(x_{1}, y_{1}\right),\left(x_{2}, y_{2}\right) \in X \times Y$, we obtain 


$$
\begin{aligned}
&\left|\mathscr{K}_{1}\left(x_{1}, y_{1}\right)(t)-\mathscr{K}_{1}\left(x_{2}, y_{2}\right)(t)\right| \\
& \leq I^{\alpha}\left|f_{x_{1}, y_{1}}-f_{x_{2}, y_{2}}\right|(b) \\
& \quad+\frac{(b-a)^{\gamma-1}}{|\Lambda| \Gamma(\gamma)}\left[\frac{(b-a)^{\gamma_{1}-1}}{\Gamma\left(\gamma_{1}\right)}\left(\sum_{i=1}^{m}\left|\theta_{i}\right| I^{\alpha_{1}+\varphi_{i}}\left|g_{x_{1}, y_{1}}-g_{x_{2}, y_{2}}\right|\left(\xi_{i}\right)+I^{\alpha}\left|f_{x_{1}, y_{1}}-f_{x_{2}, y_{2}}\right|(b)\right)\right. \\
& \quad+\left(\sum_{i=1}^{m}\left|\theta_{i}\right| \frac{\left(\xi_{i}-a\right)^{\gamma_{1}+\varphi_{i}-1}}{\Gamma\left(\gamma_{1}+\varphi_{i}\right)}\right)\left(\sum_{j=1}^{n}\left|\zeta_{j}\right| I^{\alpha+\psi_{j}}\left|f_{x_{1}, y_{1}}-f_{x_{2}, y_{2}}\right|\left(z_{j}\right)\right. \\
&\left.\left.\quad+I^{\alpha_{1}}\left|g_{x_{1}, y_{1}}-g_{x_{2}, y_{2}}\right|(b)\right)\right] \\
& \leq\left(\ell_{1}\left\|x_{1}-x_{2}\right\|+\ell_{2}\left\|y_{1}-y_{2}\right\|\right) I^{\alpha}(1)(b) \\
& \quad+\frac{(b-a)^{\gamma-1}}{|\Lambda| \Gamma(\gamma)}\left[\frac { ( b - a ) ^ { \gamma _ { 1 } - 1 } } { \Gamma ( \gamma _ { 1 } ) } \left(\left(n_{1}\left\|x_{1}-x_{2}\right\|+n_{2}\left\|y_{1}-y_{2}\right\|\right) \sum_{i=1}^{m}\left|\theta_{i}\right| I^{\alpha_{1}+\varphi_{i}}(1)\left(\xi_{i}\right)\right.\right. \\
& \quad+\left(\ell_{1}\left\|x_{1}-x_{2}\right\|+\ell_{2}\left\|y_{1}-y_{2}\right\|\right) I^{\alpha}(1)(b) \\
& \quad+\left(\ell_{1}\left\|x_{1}-x\right\|_{2}+\ell_{2}\left\|y_{1}-y_{2}\right\|\right)\left(\sum_{i=1}^{m}\left|\theta_{i}\right| \frac{\left(\xi_{i}-a\right)^{\gamma_{1}+\varphi_{i}-1}}{\Gamma\left(\gamma_{1}+\varphi_{i}\right)}\right)\left(\sum_{j=1}^{n}\left|\zeta_{j}\right| I^{\alpha+\psi_{j}}(1)\left(z_{j}\right)\right. \\
&\left.\left.+\left(n_{1}\left\|x_{1}-x\right\|_{2}+n_{2}\left\|y_{1}-y_{2}\right\|\right) I^{\alpha_{1}}(1)(b)\right)\right] \\
& \leq M_{1}\left(\ell_{1}\left\|x_{1}-x\right\|_{2}+\ell_{2}\left\|y_{1}-y_{2}\right\|\right)+M_{2}\left(n_{1}\left\|x_{1}-x\right\|_{2}+n_{2}\left\|y_{1}-y_{2}\right\|\right) \\
&=\left(M_{1} \ell_{1}+M_{2} n_{1}\right)\left\|x_{1}-x\right\|_{2}+\left(M_{1} \ell_{2}+M_{2} n_{2}\right)\left\|y_{1}-y_{2}\right\| . \\
&
\end{aligned}
$$

Therefore, we obtain the following inequality:

$$
\begin{aligned}
\left\|\mathscr{K}_{1}\left(x_{1}, y_{1}\right)-\mathscr{K}_{1}\left(x_{2}, y_{2}\right)\right\| \leq & {\left[M_{1}\left(\ell_{1}+\ell_{2}\right)+M_{2}\left(n_{1}+n_{2}\right)\right] } \\
\cdot & \left(\left\|x_{1}-x_{2}\right\|+\left\|y_{1}-y_{2}\right\|\right) .
\end{aligned}
$$

In addition, we also obtain

$$
\begin{aligned}
\left\|\mathscr{K}_{2}\left(x_{1}, y_{1}\right)-\mathscr{K}_{2}\left(x_{2}, y_{2}\right)\right\| \leq & {\left[M_{3}\left(\ell_{1}+\ell_{2}\right)+M_{4}\left(n_{1}+n_{2}\right)\right] } \\
\cdot & \left(\left\|x_{1}-x_{2}\right\|+\left\|y_{1}-y_{2}\right\|\right) .
\end{aligned}
$$

From (39) and (40), it yields

$$
\begin{aligned}
\left\|\mathscr{K}\left(x_{1}, y_{1}\right)-\mathscr{K}\left(x_{2}, y_{2}\right)\right\| \leq & {\left[\left(M_{1}+M_{3}\right)\left(\ell_{1}+\ell_{2}\right)\right.} \\
& \left.+\left(M_{2}+M_{4}\right)\left(n_{1}+n_{2}\right)\right] \\
& \times\left(\left\|x_{1}-x_{2}\right\|+\left\|y_{1}-y_{2}\right\|\right) .
\end{aligned}
$$

As $\quad\left(M_{1}+M_{3}\right)\left(\ell_{1}+\ell_{2}\right)+\left(M_{2}+M_{4}\right)\left(n_{1}+n_{2}\right)<1$, therefore, $\mathscr{K}$ is a contraction operator. By Banach's fixed point theorem, the operator $\mathscr{K}$ has a unique fixed point, which is the unique solution of (4) on $[a, b]$. The proof is completed.

Now, we prove our second existence result via Leray-Schauder alternative.
Lemma 3 (Leray-Schauder alternative, see [19]). Let $F: E \longrightarrow E$ be a completely continuous operator. Let

$\xi(F)=\{x \in E: x=\lambda F(x)$ for some, $\quad 0<\lambda<1\}$.

Then, either the set $\xi(F)$ is unbounded, or $F$ has at least one fixed point.

Theorem 2. Assume that there exist real constants $u_{i}, v_{i} \geq 0$ for $i=1,2$ and $u_{0}, v_{0}>0$ such that, for any $x_{i} \in \mathbb{R}(i=1,2)$, we have

$$
\begin{gathered}
\left|f\left(t, x_{1}, x_{2}\right)\right| \leq u_{0}+u_{1}\left|x_{1}\right|+u_{2}\left|x_{2}\right|, \\
\left|g\left(t, x_{1}, x_{2}\right)\right| \leq v_{0}+v_{1}\left|x_{1}\right|+v_{2}\left|x_{2}\right| .
\end{gathered}
$$

If $\left(M_{1}+M_{3}\right) u_{1}+\left(M_{2}+M_{4}\right) v_{1}<1$ and $\left(M_{1}+M_{3}\right) u_{2}+$ $\left(M_{2}+M_{4}\right) v_{2}<1$, where $M_{1}, M_{2}, M_{3}$, and $M_{4}$ are given in (24)-(27), then (4) has at least one solution on $[a, b]$.

Proof. By continuity of the functions $f$ and $g$ on $[a, b] \times \mathbb{R} \times \mathbb{R}$, the operator $\mathscr{K}$ is continuous. We will show that the operator $\mathscr{K}: X \times Y \longrightarrow X \times Y$ is completely continuous. Let $\Phi \subset X \times Y$ be bounded. Then, there exist positive constants $L_{1}$ and $L_{2}$ such that

$$
|f(t, x, y)| \leq L_{1},|g(t, x, y)| \leq L_{2}, \quad \forall(x, y) \in \Phi .
$$

Then, for any $(x, y) \in \Phi$, we have 


$$
\begin{aligned}
\left|\mathscr{K}_{1}(x, y)(t)\right| \leq I^{\alpha} \mid & f_{x, y} \mid(b) \\
& +\frac{(b-a)^{\gamma-1}}{|\Lambda| \Gamma(\gamma)}\left[\frac{(b-a)^{\gamma_{1}-1}}{\Gamma\left(\gamma_{1}\right)}\left(\sum_{i=1}^{m}\left|\theta_{i}\right| I^{\alpha_{1}+\varphi_{i}}\left|g_{x, y}\right|\left(\xi_{i}\right)+I^{\alpha}\left|f_{x, y}\right|(b)\right)\right. \\
& \left.+\left(\sum_{i=1}^{m}\left|\theta_{i}\right| \frac{\left(\xi_{i}-a\right)^{\gamma_{1}+\varphi_{i}-1}}{\Gamma\left(\gamma_{1}+\varphi_{i}\right)}\right)\left(\sum_{j=1}^{n}\left|\zeta_{j}\right| I^{\alpha+\psi_{j}}\left|f_{x, y}\right|\left(z_{j}\right)+I^{\alpha_{1}}\left|g_{x, y}\right|(b)\right)\right] \\
\leq & L_{1} I^{\alpha}(1)(b) \\
& +\frac{(b-a)^{\gamma-1}}{|\Lambda| \Gamma(\gamma)}\left[\frac{(b-a)^{\gamma_{1}-1}}{\Gamma\left(\gamma_{1}\right)}\left(L_{2} \sum_{i=1}^{m}\left|\theta_{i}\right| I^{\alpha_{1}+\varphi_{i}}(1)\left(\xi_{i}\right)+L_{1} I^{\alpha}(1)(b)\right)\right. \\
& \left.+\left(\sum_{i=1}^{m}\left|\theta_{i}\right| \frac{\left(\xi_{i}-a\right)^{\gamma_{1}+\varphi_{i}-1}}{\Gamma\left(\gamma_{1}+\varphi_{i}\right)}\right)\left(L_{1} \sum_{j=1}^{n}\left|\zeta_{j}\right| I^{\alpha+\psi_{j}}(1)\left(z_{j}\right)+L_{2} I^{\alpha_{1}}(1)(b)\right)\right] \\
\leq & L_{1} M_{1}+L_{2} M_{2},
\end{aligned}
$$

which yields

$$
\left\|\mathscr{K}_{1}(x, y)\right\| \leq L_{1} M_{1}+L_{2} M_{2} .
$$

Similarly, we obtain that

$$
\left\|\mathscr{K}_{2}(x, y)\right\| \leq L_{1} M_{3}+L_{2} M_{4} .
$$

Hence, from the above inequalities, we obtain that the set $\mathscr{K} \Phi$ is uniformly bounded. Next, we are going to prove that the set $\mathscr{K} \Phi$ is equicontinuous. For any $(x, y) \in \Phi$ and $\tau_{1}, \tau_{2} \in[a, b]$ such that $\tau_{1}<\tau_{2}$, we have

$$
\begin{aligned}
\left|\mathscr{K}_{1}(x, y)\left(\tau_{2}\right)-\mathscr{K}_{1}(x, y)\left(\tau_{1}\right)\right| & \\
\leq & I^{\alpha}\left|f_{x, y}\right|\left(\tau_{2}\right)-I^{\alpha}\left|f_{x, y}\right|\left(\tau_{1}\right) \\
& +\frac{\left[\left(\tau_{2}-a\right)^{\gamma-1}-\left(\tau_{1}-a\right)^{\gamma-1}\right]}{|\Lambda| \Gamma(\gamma)}\left[\frac{(b-a)^{\gamma_{1}-1}}{\Gamma\left(\gamma_{1}\right)}\left(\sum_{i=1}^{m}\left|\theta_{i}\right| I^{\alpha_{1}+\varphi_{i}}\left|g_{x, y}\right|\left(\xi_{i}\right)+I^{\alpha}\left|f_{x, y}\right|(b)\right)\right. \\
& \left.+\left(\sum_{i=1}^{m}\left|\theta_{i}\right| \frac{\left(\xi_{i}-a\right)^{\gamma_{1}+\varphi_{i}-1}}{\Gamma\left(\gamma_{1}+\varphi_{i}\right)}\right)\left(\sum_{j=1}^{n}\left|\zeta_{j}\right| I^{\alpha+\psi_{j}}\left|f_{x, y}\right|\left(z_{j}\right)+I^{\alpha_{1}}\left|g_{x, y}\right|(b)\right)\right] \\
\leq & L_{1}\left|\int_{a}^{\tau_{1}} \frac{\left(\tau_{2}-s\right)^{\alpha-1}-\left(\tau_{1}-a\right)^{\alpha-1}}{\Gamma(\alpha)} d s+\int_{\tau_{1}}^{\tau_{2}} \frac{\left(\tau_{2}-s\right)^{\alpha-1}}{\Gamma(\alpha)} d s\right| \\
& +\frac{\left[\left(\tau_{2}-a\right)^{\gamma-1}-\left(\tau_{1}-a\right)^{\gamma-1}\right]}{|\Lambda| \Gamma(\gamma)}\left[\frac{(b-a)^{\gamma_{1}-1}}{\Gamma\left(\gamma_{1}\right)}\left(L_{2} \sum_{i=1}^{m}\left|\theta_{i}\right| I^{\alpha_{1}+\varphi_{i}}(1)\left(\xi_{i}\right)+L_{1} I^{\alpha}(1)(b)\right)\right. \\
& \left.+\left(\sum_{i=1}^{m}\left|\theta_{i}\right| \frac{\left(\xi_{i}-a\right)^{\gamma_{1}+\varphi_{i}-1}}{\Gamma\left(\gamma_{1}+\varphi_{i}\right)}\right)\left(L_{1} \sum_{j=1}^{n}\left|\zeta_{j}\right|^{\alpha+\psi_{j}}(1)\left(z_{j}\right)+L_{2} I^{\alpha_{1}}(1)(b)\right)\right] \\
\leq & \frac{L_{1}}{\Gamma(\alpha+1)}\left[2\left(\tau_{2}-\tau_{1}\right)^{\alpha}+\left(\tau_{2}-a\right)^{\alpha}-\left(\tau_{1}-a\right)^{\alpha}\right] \\
& +\frac{\left[\left(\tau_{2}-a\right)^{\gamma-1}-\left(\tau_{1}-a\right)^{\gamma-1}\right]}{|\Lambda| \Gamma(\gamma)}\left[\frac{(b-a)^{\gamma_{1}-1}}{\Gamma\left(\gamma_{1}\right)}\left(L_{2} \sum_{i=1}^{m}\left|\theta_{i}\right| \frac{\left(\xi_{i}-a\right)^{\alpha_{1}+\varphi_{i}}}{\Gamma\left(\alpha_{1}+\varphi_{i}+1\right)}+L_{1} \frac{(b-a)^{\alpha}}{\Gamma(\alpha+1)}\right)\right. \\
& \left.+\left(\sum_{i=1}^{m}\left|\theta_{i}\right| \frac{\left(\xi_{i}-a\right)^{\gamma_{1}+\varphi_{i}-1}}{\Gamma\left(\gamma_{1}+\varphi_{i}\right)}\right)\left(L_{1} \sum_{j=1}^{n}\left|\zeta_{j}\right| \frac{\left(z_{j}-a\right)^{\alpha+\psi_{j}}}{\alpha+\psi_{j}+1}+L_{2} \frac{(b-a)^{\alpha_{1}}}{\Gamma(\alpha+1)}\right)\right] .
\end{aligned}
$$


Therefore, we obtain

$\left|\mathscr{K}_{1}(x, y)\left(\tau_{2}\right)-\mathscr{K}_{1}(x, y)\left(\tau_{1}\right)\right| \longrightarrow 0, \quad$ as $\tau_{1} \longrightarrow \tau_{2}$.

Analogously, we can obtain the following inequality: $\left|\mathscr{K}_{2}(x, y)\left(\tau_{2}\right)-\mathscr{K}_{2}(x, y)\left(\tau_{1}\right)\right| \longrightarrow 0, \quad$ as $\tau_{1} \longrightarrow \tau_{2}$.

Hence, the set $\mathscr{K} \Phi$ is equicontinuous. By applying the Arzelá-Ascoli theorem, the set $\mathscr{K} \Phi$ is relative compact which implies that the operator $\mathscr{K}$ is completely continuous. Lastly, we shall show that the set $\xi=\{(x, y) \in X \times Y:(x, y)=\lambda \mathscr{K}(x, y), \quad 0 \leq \lambda \leq 1\} \quad$ is bounded. Let any $(x, y) \in \xi$, then $(x, y)=\lambda \mathscr{K}(x, y)$. For any $t \in[a, b]$, we have

$$
\begin{aligned}
& x(t)=\lambda \mathscr{K}_{1}(x, y)(t), \\
& y(t)=\lambda \mathscr{K}_{2}(x, y)(t) .
\end{aligned}
$$

Then, we obtain

$$
\begin{aligned}
& \|x\| \leq\left(u_{0}+u_{1}\|x\|+u_{2}\|y\|\right) M_{1}+\left(v_{0}+v_{1}\|x\|+v_{2}\|y\|\right) M_{2}, \\
& \|y\| \leq\left(u_{0}+u_{1}\|x\|+u_{2}\|y\|\right) M_{3}+\left(v_{0}+v_{1}\|x\|+v_{2}\|y\|\right) M_{4},
\end{aligned}
$$

which imply that

$$
\begin{aligned}
\|x\|+\|y\| \leq & \left(M_{1}+M_{3}\right) u_{0}+\left(M_{2}+M_{4}\right) v_{0} \\
& +\left[\left(M_{1}+M_{3}\right) u_{1}+\left(M_{2}+M_{4}\right) v_{1}\right]\|x\| \\
& +\left[\left(M_{1}+M_{3}\right) u_{2}+\left(M_{2}+M_{4}\right) v_{2}\right]\|y\| .
\end{aligned}
$$

Thus, we obtain

$$
\|(x, y)\| \leq \frac{\left(M_{1}+M_{3}\right) u_{0}+\left(M_{2}+M_{4}\right) v_{0}}{M^{*}},
$$

where $\quad M^{*}=\min \left\{1-\left(M_{1}+M_{3}\right) u_{1}-\left(M_{2}+M_{4}\right) v_{1}\right.$, $\left.1-\left(M_{1}+M_{3}\right) u_{2}-\left(M_{2}+M_{4}\right) v_{2}\right\}$, which shows that the set $\xi$ is bounded. Therefore, by applying Lemma 3, the operator $\mathscr{K}$ has at least one fixed point. Therefore, we deduce that problem (4) has at least one solution on $[a, b]$. The proof is complete.

The last existence theorem is based on Krasnoselskii's fixed point theorem.

Lemma 4 (Krasnoselskii's fixed point theorem, see [20]). Let $M$ be a closed, bounded, convex, and nonempty subset of a Banach space $X$. Let $A$ and $B$ be operators such that (i) $A x+B y \in M$, where $x, y \in M$, (ii) $A$ is compact and continuous, and (iii) $B$ is a contraction mapping. Then, there exists $z \in M$ such that $z=A z+B z$.

Theorem 3. Assume that $f, g:[a, b] \times \mathbb{R} \times \mathbb{R} \longrightarrow \mathbb{R}$ are continuous functions satisfying assumption $\left(H_{1}\right)$ in Theorem 1. In addition, we suppose and there exist two positive constants $P, Q$ such that, for all $t \in[a, b]$ and $x_{i}, y_{i} \in \mathbb{R}, i=1,2$,

$$
\begin{gathered}
\left|f\left(t, x_{1}, x_{2}\right)\right| \leq P, \\
\left|g\left(t, x_{1}, x_{2}\right)\right| \leq Q .
\end{gathered}
$$

$$
\text { If } \frac{(b-a)^{\alpha}}{\Gamma(\alpha+1)}\left(\ell_{1}+\ell_{2}\right)+\frac{(b-a)^{\alpha_{1}}}{\Gamma(\alpha+1)}\left(n_{1}+n_{2}\right)<1,
$$

then problem (4) has at least one solution on $[a, b]$.

Proof. To apply Lemma 4, we decompose the operator $\mathscr{K}$ into four operators $\mathscr{K}_{1,1}, \mathscr{K}_{1,2}, \mathscr{K}_{2,1}$, and $\mathscr{K}_{2,2}$ as

$$
\begin{aligned}
\mathscr{K}_{1,1}(x, y)(t)= & \frac{(t-a)^{\gamma-1}}{\Lambda \Gamma(\gamma)}\left[\frac{(b-a)^{\gamma_{1}-1}}{\Gamma\left(\gamma_{1}\right)}\left(\sum_{i=1}^{m} \theta_{i} I^{\alpha_{1}+\varphi_{i}} g_{x, y}\left(\xi_{i}\right)-I^{\alpha} f_{x, y}(b)\right)\right. \\
& \left.+\left(\sum_{i=1}^{m} \theta_{i} \frac{\left(\xi_{i}-a\right)^{\gamma_{1}+\varphi_{i}-1}}{\Gamma\left(\gamma_{1}+\varphi_{i}\right)}\right)\left(\sum_{j=1}^{n} \zeta_{j} I^{\alpha+\psi_{j}} f_{x, y}\left(z_{j}\right)-I^{\alpha_{1}} g_{x, y}(b)\right)\right], \\
\mathscr{K}_{1,2}(x, y)(t)= & I^{\alpha} f_{x, y}(t), \\
\mathscr{K}_{2,1}(x, y)(t)= & \frac{(t-a)^{\gamma_{1}-1}}{\Lambda \Gamma\left(\gamma_{1}\right)}\left[\frac{(b-a)^{\gamma-1}}{\Gamma(\gamma)}\left(\sum_{j=1}^{n} \zeta_{j} I^{\alpha+\psi_{j}} f_{x, y}\left(z_{j}\right)-I^{\alpha_{1}} g_{x, y}(b)\right)\right. \\
& \left.+\left(\sum_{j=1}^{n} \zeta_{j} \frac{\left(z_{j}-a\right)^{\gamma+\psi_{j}-1}}{\Gamma\left(\gamma+\psi_{j}\right)}\right)\left(\sum_{i=1}^{m} \theta_{i} I^{\alpha_{1}+\varphi_{i}} g_{x, y}\left(\xi_{i}\right)-I^{\alpha} f_{x, y}(b)\right)\right] \\
\mathscr{K}_{2,2}(x, y)(t)= & I^{\alpha_{1}} g_{x, y}(t) .
\end{aligned}
$$


Note that $\mathscr{K}_{1}(x, y)(t)=\mathscr{K}_{1,1}(x, y)(t)+\mathscr{K}_{1,2}(x, y)(t)$ and $\quad \mathscr{K}_{2}(x, y)(t)=\mathscr{K}_{2,1}(x, y)(t)+\mathscr{K}_{2,2}(x, y)(t)$. Also, observe that the ball $B_{\delta}$ is a closed, bounded, and convex subset of the Banach space $\mathscr{C}$. Let $B_{\delta}=\{(x, y) \in X \times$ $Y:\|(x, y)\| \leq \delta\}$ be a ball, where a constant $\delta \geq \max \left\{M_{1} P+\right.$ $\left.M_{2} Q, M_{3} P+M_{4} Q\right\}$. Now, we will show that $\mathscr{K} B_{\delta} \subset B_{\delta}$ for satisfying condition (i) of Lemma 4. Setting $x=$ $\left(x_{1}, x_{2}\right)$ and $y=\left(y_{1}, y_{2}\right) \in B_{\delta}$ and using condition (55), then we have, as in Theorem 2 that

$$
\left|\mathscr{K}_{1,1}\left(x_{1}, x_{2}\right)(t)+\mathscr{K}_{1,2}\left(y_{1}, y_{2}\right)(t)\right| \leq M_{1} P+M_{2} Q \leq \delta .
$$

Similarly, we can find that

$$
\left|\mathscr{K}_{2,1}\left(x_{1}, x_{2}\right)(t)+\mathscr{K}_{2,2}\left(y_{1}, y_{2}\right)(t)\right| \leq M_{3} P+M_{4} Q \leq \delta .
$$

That yields $\mathscr{K}_{1} x+\mathscr{K}_{2} y \in B_{\delta}$. To show that the operator $\left(\mathscr{K}_{1,2}, \mathscr{K}_{2,2}\right)$ is a contraction mapping satisfying condition (iii) of Lemma 4, for $\left(x_{1}, y_{1}\right),\left(x_{2}, y_{2}\right) \in B_{\delta}$, we have

$$
\begin{aligned}
& \left|\mathscr{K}_{1,2}\left(x_{1}, y_{1}\right)\right|(t)-\mathscr{K}_{1,2}\left(x_{2}, y_{2}\right)(t)\left|\leq I^{\alpha} \| f_{x_{1}, y_{1}}-f_{x_{2}, y_{2}}\right|(t) \\
& \quad \leq\left(\ell_{1}\left\|x_{1}-x_{2}\right\|+\ell_{2}\left\|y_{1}-y_{2}\right\|\right) I^{\alpha}(1)(b) \\
& \quad \leq \frac{(b-a)^{\alpha}}{\Gamma(\alpha+1)}\left(\ell_{1}+\ell_{2}\right)\left(\left\|x_{1}-x_{2}\right\|+\left\|y_{1}-y_{2}\right\|\right) \\
& \left|\mathscr{K}_{2,2}\left(x_{1}, y_{1}\right)(t)-\mathscr{K}_{2,2}\left(x_{2}, y_{2}\right)(t)\right| \leq I^{\alpha_{1}}\left|g_{x_{1}, y_{1}}-g_{x_{2}, y_{2}}\right|(t) \\
& \quad \leq \frac{(b-a)^{\alpha_{1}}}{\Gamma\left(\alpha_{1}+1\right)}\left(n_{1}+n_{2}\right)\left(\left\|x_{1}-x_{2}\right\|+\left\|y_{1}-y_{2}\right\|\right) .
\end{aligned}
$$

It follows from (60) and (61) that

$$
\begin{aligned}
\left\|\left(\mathscr{K}_{1,2}, \mathscr{K}_{2,2}\right)\left(x_{1}, y_{1}\right)-\left(\mathscr{K}_{1,2}, \mathscr{K}_{2,2}\right)\left(x_{2}, y_{2}\right)\right\| \\
\leq\left[\frac{(b-a)^{\alpha}}{\Gamma(\alpha+1)}\left(\ell_{1}+\ell_{2}\right)+\frac{(b-a)^{\alpha_{1}}}{\Gamma\left(\alpha_{1}+1\right)}\left(n_{1}+n_{2}\right)\right] \\
\cdot\left(\left\|x_{1}-x_{2}\right\|+\left\|y_{1}-y_{2}\right\|\right),
\end{aligned}
$$

which is a contraction by inequality in (56). Therefore, condition (iii) of Lemma 4 is satisfied. Next, we will show that the operator $\left(\mathscr{K}_{1,1}, \mathscr{K}_{2,1}\right)$ satisfies condition (ii) of Lemma 4 . By applying the continuity of the functions $f, g$ on $[a, b] \times \mathbb{R} \times \mathbb{R}$, we can conclude that the operator $\left(\mathscr{K}_{1,1}, \mathscr{K}_{2,1}\right)$ is continuous. For each $(x, y) \in B_{\delta}$, one has

$$
\begin{aligned}
\left|\mathscr{K}_{1,1}(x, y)(t)\right| & \\
\leq & \frac{(b-a)^{\gamma-1}}{|\Lambda| \Gamma(\gamma)}\left[\frac{(b-a)^{\gamma_{1}-1}}{\Gamma\left(\gamma_{1}\right)}\left(Q \sum_{i=1}^{m}\left|\theta_{i}\right| I^{\alpha_{1}+\varphi_{i}}(1)\left(\xi_{i}\right)+P I^{\alpha}(1)(b)\right)\right. \\
& \left.+\left(\sum_{i=1}^{m}\left|\theta_{i}\right| \frac{\left(\xi_{i}-a\right)^{\gamma_{1}+\varphi_{i}-1}}{\Gamma\left(\gamma_{1}+\varphi_{i}\right)}\right)\left(P \sum_{j=1}^{n}\left|\zeta_{j}\right| I^{\alpha+\psi_{j}}(1)\left(z_{j}\right)+Q I^{\alpha_{1}}(1)(b)\right)\right] \\
= & \frac{(b-a)^{\gamma-1}}{|\Lambda| \Gamma(\gamma)}\left[\frac{(b-a)^{\gamma_{1}-1}}{\Gamma\left(\gamma_{1}\right)}\left(Q \sum_{i=1}^{m}\left|\theta_{i}\right| \frac{\left(\xi_{i}-a\right)^{\alpha_{1}+\varphi_{i}}}{\Gamma\left(\alpha_{1}+\varphi_{i}+1\right)}+P \frac{(b-a)^{\alpha}}{\Gamma(\alpha+1)}\right)\right. \\
& \left.+\left(\sum_{i=1}^{m}\left|\theta_{i}\right| \frac{\left(\xi_{i}-a\right)^{\gamma_{1}+\varphi_{i}-1}}{\Gamma\left(\gamma_{1}+\varphi_{i}\right)}\right)\left(P \sum_{j=1}^{n}\left|\zeta_{j}\right| \frac{\left(z_{j}-a\right)^{\alpha+\psi_{j}}}{\Gamma\left(\alpha+\psi_{j}+1\right)}+Q \frac{(b-a)^{\alpha_{1}}}{\Gamma\left(\alpha_{1}+1\right)}\right)\right] \\
:= & P^{*},
\end{aligned}
$$


and similarly

$$
\left|\mathscr{K}_{2,1}(x, y)(t)\right| \leq Q^{*} \text {. }
$$

Then, we obtain the following fact:

$$
\left\|\left(\mathscr{K}_{1,1}, \mathscr{K}_{2,1}\right)(x, y)\right\| \leq P^{*}+Q^{*},
$$

which implies that the set $\left(\mathscr{K}_{1,1}, \mathscr{K}_{2,1}\right) B_{\delta}$ is uniformly bounded. In the next step, we will show that the set $\left(\mathscr{K}_{1,1}, \mathscr{K}_{2,1}\right) B_{\delta}$ is equicontinuous. For $\tau_{1}, \tau_{2} \in[a, b]$ such that $\tau_{1}<\tau_{2}$ and for any $(x, y) \in B_{\delta}$, we can prove that

$$
\begin{aligned}
\left|\mathscr{K}_{1,1}(x, y)\left(\tau_{2}\right)-\mathscr{K}_{1,1}(x, y)\left(\tau_{1}\right)\right| & \\
\leq & \frac{\left[\left(\tau_{2}-a\right)^{\gamma-1}-\left(\tau_{1}-a\right)^{\gamma-1}\right]}{|\Lambda| \Gamma(\gamma)}\left[\frac{(b-a)^{\gamma_{1}-1}}{\Gamma\left(\tau_{1}\right)}\left(Q \sum_{i=1}^{m}\left|\theta_{i}\right| I^{\alpha_{1}+\varphi_{i}}(1)\left(\xi_{i}\right)+P I(1)(b)\right)\right. \\
& \left.+\left(\sum_{i=1}^{m}\left|\theta_{i}\right| \frac{\left(\xi_{i}-a\right)^{\gamma_{1}+\varphi_{i}-1}}{\Gamma\left(\gamma_{1}+\varphi_{i}\right)}\right)\left(P \sum_{j=1}^{n}\left|\zeta_{j}\right| I^{\alpha+\psi_{j}}(1)\left(z_{j}\right)+Q I^{\alpha_{1}}(1)(b)\right)\right] \\
\leq & \frac{\left[\left(\tau_{2}-a\right)^{\gamma-1}-\left(\tau_{1}-a\right)^{\gamma-1}\right]}{|\Lambda| \Gamma(\gamma)}\left[\frac{(b-a)^{\gamma_{1}-1}}{\Gamma\left(\gamma_{1}\right)}\left(Q \sum_{i=1}^{m}\left|\theta_{i}\right| \frac{\left(\xi_{i}-a\right)^{\alpha_{1}+\varphi_{i}}}{\Gamma\left(\alpha_{1}+\varphi_{i}+1\right)}+P \frac{(b-a)^{\alpha}}{\Gamma(\alpha+1)}\right)\right. \\
& \left.+\left(\sum_{i=1}^{m}\left|\theta_{i}\right| \frac{\left(\xi_{i}-a\right)^{\gamma_{1}+\varphi_{i}-1}}{\Gamma\left(\gamma_{1}+\varphi_{i}\right)}\right)\left(P \sum_{j=1}^{n}\left|\zeta_{j}\right| \frac{\left(z_{j}-a\right)^{\alpha+\psi_{j}}}{\Gamma\left(\alpha+\psi_{j}+1\right)}+Q \frac{(b-a)^{\alpha_{1}}}{\Gamma\left(\alpha_{1}+1\right)}\right)\right] .
\end{aligned}
$$

Therefore, we have $\mid \mathscr{K}_{1,1}(x, y)\left(\tau_{2}\right)-\mathscr{K}_{1,1}(x, y)$ $\left(\tau_{1}\right) \mid \longrightarrow 0$ as $\tau_{1} \longrightarrow \tau_{2}$. Similarly, we can show that $\left|\mathscr{K}_{2,1}(x, y)\left(\tau_{2}\right)-\mathscr{K}_{2,1}(x, y)\left(\tau_{1}\right)\right| \longrightarrow 0$ as $\tau_{1} \longrightarrow \tau_{2}$.

Thus, $\left|\left(\mathscr{K}_{1,1}, \mathscr{K}_{2,1}\right)(x, y)\left(\tau_{2}\right)-\left(\mathscr{K}_{1,1}, \mathscr{K}_{2,1}\right)(x, y)\left(\tau_{1}\right)\right|$ tends to zero as $\tau_{1} \longrightarrow \tau_{2}$. Therefore, the set $\left(\mathscr{K}_{1,1}, \mathscr{K}_{2,1}\right) B_{\delta}$ is equicontinuous. By applying the Arzelá-Ascoli theorem, the operator $\left(\mathscr{K}_{1,1}, \mathscr{K}_{2,1}\right)$ is compact on $B_{\delta}$. By application of Lemma 4, we have that problem (4) has at least one solution on $[a, b]$. This completes the proof.

Example 1. Consider the coupled system of Hilfer fractional differential equations with nonlocal integral boundary conditions of the form

$$
\left\{\begin{array}{l}
{ }^{H} D^{(3 / 2),(1 / 2)} x(t)=f(t, x(t), y(t)), \quad t \in\left[\frac{1}{3}, \frac{10}{3}\right], \\
{ }^{H} D^{(5 / 4),(2 / 3)} y(t)=g(t, x(t), y(t)), \quad t \in\left[\frac{1}{3}, \frac{10}{3}\right], \\
x\left(\frac{1}{3}\right)=0, \quad x\left(\frac{10}{3}\right)=\frac{1}{2} I^{1 / 3} y\left(\frac{2}{3}\right)+\frac{2}{3} I^{1 / 2} y(1)+\frac{3}{4} I^{3 / 5} y\left(\frac{4}{3}\right)+\frac{4}{5} I^{2 / 3} y\left(\frac{5}{3}\right), \\
y\left(\frac{1}{3}\right)=0, \quad y\left(\frac{10}{3}\right)=\frac{3}{2} I^{3 / 2} x(2)+\frac{4}{3} I^{5 / 3} x\left(\frac{7}{3}\right)+\frac{5}{4} I^{7 / 4} y\left(\frac{8}{3}\right)+\frac{6}{5} I^{9 / 5} x(3) .
\end{array}\right.
$$

Here, $\alpha=3 / 2, \quad \alpha_{1}=5 / 4, \quad \beta=1 / 2, \quad \beta_{1}=2 / 3, \quad a=1 / 3$, $b=10 / 3, m=n=4, \theta_{i}=(i /(i+1)), \quad \zeta_{i}=((2+i) /(1+i))$, $\varphi_{i}=(i /(i+2))$, and $\psi_{i}=((2 i+1) /(i+1)), \quad i=1,2,3,4$, $\xi_{j}=(j / 3), j=2,3,4,5$, and $z_{r}=(r / 3), r=6,7,8,9$. Then, we can compute constants as $\gamma=7 / 4, \quad \gamma_{1}=41 / 21$, $\Lambda=6.371398411, \quad M_{1}=12.56809051, M_{2}=3.253588460$, $M_{3}=48.72536839$, and $M_{4}=22.05071608$.

(i) Let the nonlinear functions $f$ and $g$ be defined by 
$f(t, x, y)=\frac{9 e^{-t}}{9 t^{2}+3239}\left(\frac{x^{2}+2|x|}{1+|x|}\right)+\frac{3}{3 t+539} \sin |y|+\frac{2}{3}$,

$g(t, x, y)=\frac{9}{10(3 t+11)^{2}} \tan ^{-1}|x|+\left(\frac{3 \cos ^{2} t}{479+3 t}\right) \frac{|y|}{1+|y|}+\frac{1}{2}$

It is easy to check that $f$ and $g$ satisfy $\left(H_{1}\right)$ in Theorem 1 as $\left|f\left(t, x_{1}, y_{1}\right)-f\left(t, x_{2}, y_{2}\right)\right| \leq(1 / 180) \mid x_{1}$ $-x_{2}|+(1 / 180)| y_{1}-y_{2} \mid$ and $\mid g\left(t, x_{1}, y_{1}\right)-g\left(t, x_{2}\right.$, $\left.y_{2}\right)|\leq(1 / 160)| x_{1}-x_{2}|+(1 / 160)| y_{1}-y_{2} \mid$. Setting $\ell_{1}=\ell_{2}=(1 / 180)$ and $n_{1}=n_{2}=(1 / 160)$, we have

$\left(M_{1}+M_{3}\right)\left(\ell_{1}+\ell_{2}\right)+\left(M_{2}+M_{4}\right)\left(n_{1}+n_{2}\right)=0.9973422390<1$.

Therefore, by applying Theorem 1, we deduce that problem (67) with (68)-(69) has a unique solution $(x, y)$ on $[1 / 3,10 / 3]$.

(ii) Given the functions $f$ and $g$ by

$f(t, x, y)=\frac{3 e^{-|y|}}{5(3 t+50)}\left(\frac{|x|^{9}}{1+x^{8}}\right)+\frac{9}{(3 t+29)^{2}}\left(\frac{y^{4}}{1+|y|^{3}}\right)+\frac{3}{4}$,

$g(t, x, y)=\frac{3 e^{-y^{2}} \sin |x|}{\left(19+x^{2}\right)(14+3 t)}+\frac{3 y \cos ^{2}(x y)}{4(50+3 t)}+\frac{4}{7}$,

we can check that

$$
\begin{aligned}
& |f(t, x, y)| \leq \frac{3}{4}+\frac{1}{85}|x|+\frac{1}{100}|y|, \\
& |g(t, x, y)| \leq \frac{4}{7}+\frac{1}{95}|x|+\frac{1}{68}|y| .
\end{aligned}
$$

which satisfy conditions in Theorem 2 by $u_{0}=3 / 4$, $v_{0}=4 / 7, \quad u_{1}=1 / 85, \quad v_{1}=1 / 95, \quad u_{2}=1 / 100, \quad$ and $v_{2}=1 / 68$. Hence, we find that $\left(M_{1}+M_{3}\right) u_{1}+$ $\left(M_{2}+M_{4}\right) v_{1}=0.9874606169<1$ and $\left(M_{1}+\right.$ $\left.M_{3}\right) u_{2}+\left(M_{2}+M_{4}\right) v_{2}=0.9850567146<1$. The conclusion of Theorem 2 implies that problem (67) with (71)-(72) has at least one solution $(x, y)$ on $[1 / 3,10 / 3]$.

(iii) Define the functions $f$ and $g$ by

$f(t, x, y)=\frac{3 e^{-t^{2}}}{3 t+29}\left(\frac{|x|}{1+|x|}\right)+\frac{9}{9 t^{2}+134} \sin |y|+\frac{2}{3}$,

$$
g(t, x, y)=\frac{9}{5(3 t+5)^{2}} \tan ^{-1}|x|+\left(\frac{9}{(3 t+14)^{2}}\right)\left(\frac{|y|}{1+|y|}\right)+\frac{1}{2} .
$$

Observe that condition $\left(H_{1}\right)$ in Theorem 1 is satisfied for nonlinear functions $f$ and $g$ with Lipschitz constants $\ell_{1}=1 / 10, \ell_{2}=1 / 15, n_{1}=1 / 20$, and $n_{2}=1 / 25$. Next, we can find that $\left(M_{1}+M_{3}\right)\left(\ell_{1}+\ell_{2}\right)+\left(M_{2}+\right.$ $\left.M_{4}\right)\left(n_{1}+n_{2}\right)=12.49296389>1$. Then, Theorem 1 cannot be used to obtain the existence criteria for the investigated problem. However, we calculate that $|f(t, x, y)| \leq 5 / 6,|g(t, x, y)| \leq(108+5 \pi) / 200$, and

$$
\begin{aligned}
& \frac{(b-a)^{\alpha}}{\Gamma(\alpha+1)}\left(\ell_{1}+\ell_{2}\right)+\frac{(b-a)^{\alpha_{1}}}{\Gamma\left(\alpha_{1}+1\right)}\left(n_{1}+n_{2}\right) \\
& \quad=0.9650966816<1 .
\end{aligned}
$$

Hence, all assumptions of Theorem 3 hold. Therefore, by Theorem 3, problem (67) with (74)-(75) has at least one solution $(x, y)$ on $[1 / 3,10 / 3]$.

\section{Data Availability}

No data were used to support this study.

\section{Conflicts of Interest}

The authors declare that there are no conflicts of interest regarding the publication of this paper.

\section{Acknowledgments}

This research was funded by King Mongkut's University of Technology North Bangkok (Contract no. KMUTNB-61KNOW-031).

\section{References}

[1] K. Diethelm, "The analysis of fractional differential equations," in Lecture Notes in Mathematics, Springer, New York, NY, USA, 2010.

[2] A. A. Kilbas, H. M. Srivastava, and J. J. Trujillo, "Theory and applications of the fractional differential equations," in NorthHolland Mathematics Studies, Elsevier, Amsterdam, Netherlands, 2006.

[3] V. Lakshmikantham, S. Leela, and J. V. Devi, Theory of Fractional Dynamic Systems, Cambridge Scientific Publishers, Cambridge, England, 2009.

[4] K. S. Miller and B. Ross, An Introduction to the Fractional Calculus and Differential Equations, John Wiley, NewYork, NY, USA, 1993.

[5] I. Podlubny, Fractional Differential Equations, Academic Press, New York, NY, USA, 1999.

[6] S. G. Samko, A. A. Kilbas, and O. I. Marichev, Fractional Integrals and Derivatives, Gordon and Breach Science, Yverdon-les-Bains, Switzerland, 1993.

[7] Y. Zhou, Basic Theory of Fractional Differential Equations, World Scientific, Singapore, 2014.

[8] R. Hilfer, Applications of Fractional Calculus in Physics, World Scientific, Singapore, 2000. 
[9] R. Hilfer, "Experimental evidence for fractional time evolution in glass forming materials," Chemical Physics, vol. 284, no. 1-2, pp. 399-408, 2002.

[10] R. Hilfer, Y. Luchko, and Z. Tomovski, "Operational method for the solution of fractional differential equations with generalized Riemann-Liouvill fractional derivatives," Fractional Calculus and Applied Analysis, vol. 12, pp. 299-318, 2009.

[11] K. M. Furati, M. D. Kassim, and N. E. Tatar, "Existence and uniqueness for a problem involving Hilfer fractional derivative," Computers \& Mathematics with Applications, vol. 64, no. 6, pp. 1616-1626, 2012.

[12] H. Gu and J. J. Trujillo, "Existence of mild solution for evolution equation with Hilfer fractional derivative," Applied Mathematics and Computation, vol. 257, pp. 344-354, 2015.

[13] R. Subashini and C. Ravichandran, "On the results of nonlocal Hilfer fractional semilinear differential inclusions," Proceedings of the Jangjeon Mathematical Society, vol. 22, no. 2, pp. 249-267, 2019.

[14] R. Subashini, K. Jothimani, S. Saranya, and C. Ravichandran, "On the results of Hilfer fractional derivative with nonlocal conditions," International Journal of Pure and Applied Mathematics, vol. 118, no. 11, pp. 277-289, 2018.

[15] R. Subashini, C. Ravichandran, C. Ravichandran, K. Jothimani, and H. Mehmet Baskonus, "Existence results of Hilfer integro-differential equations with fractional order," Discrete \& Continuous Dynamical Systems-S, vol. 13, no. 3, pp. 911-923, 2020.

[16] J. Wang and Y. Zhang, "Nonlocal initial value problems for differential equations with Hilfer fractional derivative," $A p$ plied Mathematics and Computation, vol. 266, pp. 850-859, 2015.

[17] S. Asawasamrit, A. Kijjathanakorn, S. K. Ntouyas, and J. Tariboon, "Nonlocal boundary value problems for Hilfer fractional differential equations," Bulletin of the Korean Mathematical Society, vol. 55, no. 6, pp. 1639-1657, 2018.

[18] J. Vanterler da, C. Sousa, and E. Capelas de Oliveira, "On the $\psi$-Hilfer fractional derivative," Communications in Nonlinear Science and Numerical Simulation, vol. 60, pp. 72-91, 2018.

[19] A. Granas and J. Dugundji, Fixed Point Theory, SpringerVerlag, New York, NY, USA, 2003.

[20] M. A. Krasnoselskii, "Two remarks on the method of successive approximations," Uspekhi Matematicheskikh Nauk, vol. 10, pp. 123-127, 1955. 